Z. Epileptol. $2020 \cdot 33: 101-106$
https://doi.org/10.1007/s10309-020-00332-w

○ Der/die Autor(en) 2020

\author{
Adam Strzelczyk ${ }^{1}$ Friedhelm C. Schmitt ${ }^{2}$ \\ 'Epilepsiezentrum Frankfurt Rhein-Main, Zentrum der Neurologie und Neurochirurgie, Goethe- \\ Universität und Universitätsklinikum Frankfurt, Frankfurt am Main, Deutschland \\ ${ }^{2}$ Universitätsklinik für Neurologie, Otto-von-Guericke-Universität, Magdeburg, Deutschland
}

\title{
100 Jahre Dieter Janz
}

Am 20.04.2020 jährt sich zum 100. Mal der Geburtstag von Herrn Prof. emeritus Dr. med. Wolfgang Dietrich Janz $\left({ }^{\star} 20\right.$. April 1920 in Speyer, $\uparrow 25$. Dezember 2016 in Berlin) [1,2].

Aus diesem Anlass möchten wir mit diesem Jubiläumsheft Dieter Janz eine besondere Ehrung zukommen lassen (-Abb. 1) und haben zum einen Wissenschaftler und Wissenschaftlerinnen, die sich mit dem nach ihm benannten Janz-Syndrom (juvenile myoklonische Epilepsie, JME) beschäftigen, und zum anderen Mitarbeiter ( $\bullet$ Abb. 2), Freunde und langjährige Wegbegleiter gebeten, Beiträge, die sich mit dem Wirken Janz' beschäftigen, $\mathrm{zu}$ verfassen. Es handelt sich dabei nicht um zwei distinkte Gruppen, da Dieter Janz bis zu seinen letzten Tagen im Leben immer aktiv an einem wissenschaftlichen Austausch, dem Publizieren und der Weiterverbreitung seiner Sichtweisen mitgewirkt hat.

Dieses Heft beginnt mit einem Beitrag von Peter Wolf aus Dianalund (Dänemark) mit der Frage: „Wer war eigentlich Dieter Janz?"', in dem er anschaulich und freundschaftlich das Wirken, die Motivation und auch die charakterlichen Züge von Dieter Janz beschreibt [3]. Viele Leser hatten das Glück, Dieter Janz gut zu kennen oder ihm auf seinen zahlreichen Wirkstätten zu begegnen. Sie werden in der Beschreibung von Peter Wolf die Person Dieter Janz gut wiedererkennen können. Peter Wolf beschreibt auch das Wirken Dieter Janz' während seine Heidelberger (bis 1972) und seiner Berliner Zeit (1972-1988) als Hochschullehrer und Abteilungs- bzw. Klinikleiter (- Abb. 3, 4 und 5).

Ein großes Anliegen von Dieter Janz war auch die Stiftung Michael, so lag es nahe, Heinz Bühler aus Bonn zu bitten, einen Artikel über die Entstehung der Stiftung Michael und den Austausch mit Dieter Janz über mehrere Jahrzehnte niederzuschreiben [4]. Die Stiftung Michael ist ein Glücksfall für die Epileptologie in Deutschland und hat in den letzten Jahrzehnten zunehmendes internationales Renommee gewonnen (• Abb.6). Das Bild auf dem Cover dieses Jubiläumsheftes zeigt Dieter Janz bei seiner Rede zum 50-jährigen Bestehen der Stiftung Michael am 06.10.2012 in der Berliner Mendelssohn-Remise.

Der Michael-Preis gehört zu den herausragenden Auszeichnungen für Epileptologen auf internationalem Niveau. Dieser Artikel wird so auch jungen Nachwuchswissenschaftlern die Ursprünge, Entwicklung und Ziele der Stiftung näherbringen. Verbunden mit der Stiftung Michael und Dieter Janz sind zudem die Praxisseminare Epilepsie und EEG in Gargnano am Gardasee (Italien), die er gerne vor Ort mitgestaltete (• Abb. 7).

Dieter Janz war in der Neurologischen Klinik der Universitätsklinik Heidelberg unter der Leitung von Paul Vogel tätig. Paul Vogel wiederum war ein Schüler von Viktor von Weizsäcker, welcher seit 1920 dieselbe Neurologische Klinik leitete, bevor er der Nachfolger von Otfrid Förster in Breslau wurde. Nach 1945 leitete Viktor von Weizsäcker wieder die "Allgemeine Klinische Medizin“ in Heidelberg. Er prägte von dort wesentlich eine psychosomatische Denkweise in der Medizin und insbesondere in der Neurologie. Auch Dieter Janz ließ dies in seine Fachdisziplin einfließen. Mit diesem „Heidelberger" Hintergrund war es nur konsequent, dass er ein Mitbegründer der „Viktor-von-Weizsäcker-Gesellschaft" wurde (• Abb. 8).
Das Wirken Dieter Janz' war auch von künstlerischen und philosophischen Fragen geprägt: So publizierte er seine Interpretation der Transfiguration von Raphael in Epilepsia [5]. In diesem Zusammenhang war es eine glückliche Fügung, dass wir von Hartwig Wiedebach aus Göppingen, einem Vorstandsmitglied der „Viktor-von-Weizsäcker-Gesellschaft", einen Artikel zum 100. Geburtstag von Dieter Janz und zu eben diesem Aspekt seines Wirkens erhalten haben. In diesem Artikel werden u. a. die Sicht von Dieter Janz auf die Erkrankung und der Zugang zur Epilepsie sowie die Auseinandersetzung mit literarischen Vorbildern wiedergegeben [6].

Dieter Janz und Walter Christian veröffentlichten 1957 eine detaillierte Be-

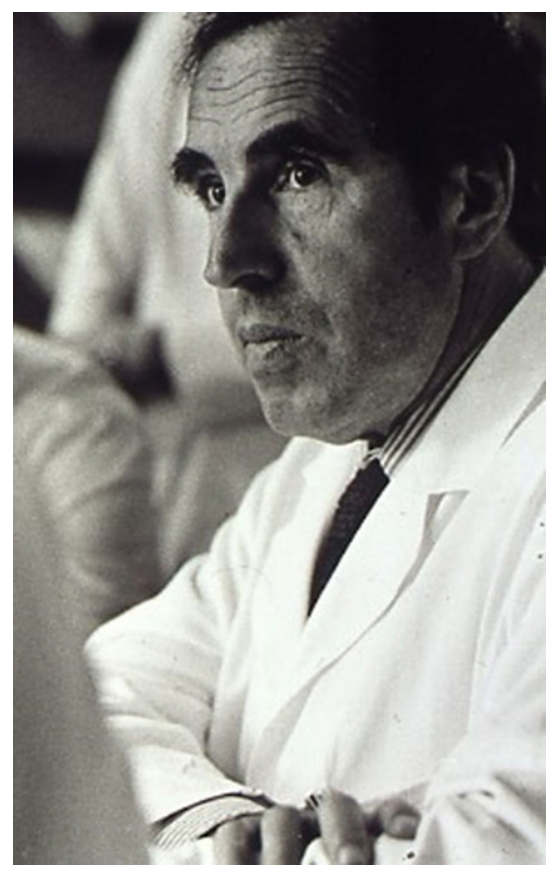

Abb. 1 A Janz bei Festakt zum 10-jährigen Bestehen der Abteilung 1983. (Foto:WalterChriste, Berlin) 
schreibung der juvenilen myoklonischen Epilepsie (JME) [7] und begründeten somit eine genaue syndromale Beschreibung und Zuordenbarkeit. Möglicherweise hat die Publikation in deutscher Sprache dazu geführt, dass eine Anerkennung als eigenständiges Syndrom international zunächst auf sich warten ließ und dass die Bezeichnung „JanzSyndrom" für die JME nur im deutschsprachigen Raum breiter verwendet wird [8]. Der Frage, wann, mit welchem Begriff und in welchem Zusammenhang Patienten mit Myoklonien und wahrscheinlich der Diagnose eines Janz-Syndroms beschrieben wurden, widmete sich Günter Krämer aus Zürich (Schweiz) in seinem Beitrag zu der Geschichte der Epilepsien mit myoklonischen Anfällen bis hin zur Beschreibung des Janz-Syndroms [9]. Die historischen Verfasser werden eingeführt, und die Krankengeschichten werden detailgenau wiedergegeben. Die Besonderheit dieser wegweisenden Publikation mit dem Namen „ImpulsivPetit Mal“ und ihre anschließende Rezeption [7] werden erörtert. Ein Faksimile des Originalartikels aus der Deutschen Zeitschrift für Nervenheilkunde wurde 2005 anlässlich des 50. Geburtstags der Originalpublikation und des 85 . Geburtstag von Dieter Janz in der Zeitschrift für Epileptologie nachgedruckt und kann online abgerufen werden [10]. Das Editorial zu dieser Ausgabe [11] schrieben Jürgen Bauer aus Bonn und Wilhelm Rimpau [11, 12] aus Berlin, Letzterer war einer der fünf ärztlichen Mitarbeiter, die Dieter Janz von Heidelberg mit an die Charlottenburger Klinik folgten. Die anderen 4 Mitarbeiter waren: Karl-Friedrich Masuhr [13, 14], HansWolfgang Kölmel [15], Dieter Schmidt [16] und Peter Wolf $[2,3,17]$. In Berlin wurden dann unter anderem Friedhelm Lamprecht (ab 1974), Gisela Schüler (ab 1974) [18], Mechthilde Kütemeyer (ab 1975) [14], Rupprecht Thorbecke (ab 1975) [18-20], Gertrud Beck-Mannagetta (ab 1976) [21-23], Friedrich Behse (ab 1977) und Hans-Joachim Meencke (ab 1979) [24, 25] seine Mitarbeiter. Mit Letzterem publizierte Dieter Janz zur Neuropathologie der primär generalisierten Epilepsien (nach heutiger Klassifikation: genetische generalisierte
Epilepsien [26, 27]). Diese Publikationen $[24,25]$ bildeten das strukturelle Pendant zu seiner klinisch-phänomenologischen Beschreibung des „Impulsiv-Petit Mal“, im Verlauf folgten weitere Publikationen $\mathrm{zu}$ den genetischen Ursachen der JME [21-23].

Das Janz-Syndrom ist inzwischen gut bekannt und klinisch eindeutig abgrenzbar. Wichtige Aussagen zu der Langzeitprognose ließen sich v. a. aus dem sog. Janz-Archiv ableiten, in dem über Jahrzehnte Patienten nachverfolgt wurden, die er in Heidelberg und in West-Berlin behandelte. Bernd Vorderwülbecke und Martin Holtkamp aus Berlin widmen sich in ihrem Artikel der Frage, wie die langfristige Prognose bezüglich der Anfallsfreiheit aussieht [28]. An den Auswertungen der klinischen Daten seines Archives hat noch Dieter Janz bis ins hohe Alter mitgewirkt (• Abb. 9), diese sind teilweise erst posthum publiziert worden [29-31]. Aus den Ergebnissen zur Langzeitprognose stellt sich konsequenterweise auch die Frage, wie lange eine Therapie mit Antiepileptika erfolgen sollte. Gerd Kurlemann aus Münster und Günter Krämer haben schon häufig auf Tagungen kontroverse und sehr unterhaltsame Diskussionen zu dieser Frage geführt, wann der richtige Zeitpunkt zum Absetzen wäre, und wir freuen uns sehr, dass sie sich bereit erklärt haben, diese Argumente auch noch mal für dieses Jubiläumsheft zusammenzutragen [32]. Die Leser werden viele Argumente für das Für und Wider des Absetzens im Beitrag wiederfinden, die auch jedem von uns in der klinischen Praxis begegnen.

Im Verständnis von Dieter Janz waren sozialmedizinische Aspekte ein integraler Bestandteil in der klinischen Versorgung von Epilepsiepatienten. Rupprecht Thorbecke aus Bielefeld gibt - als ehemaliger Mitarbeiter von Dieter Janz einen Einblick in das sozialmedizinische Wirken in der Janz'schen Klinik [19]. Bereits 2017 hat Rupprecht Thorbecke einen Artikel zu Rehabilitation als „konstituierendes Moment der epileptologischen Arbeit" von Dieter Janz in der Zeitschrift für Epileptologie veröffentlicht [20]. Neben der Langzeitanfallsprognose und der Frage der antiepileptischen Therapie ist auch die Frage, wie sich die psychosozia-
Z. Epileptol. 2020 33:101-106

https://doi.org/10.1007/s10309-020-00332-w

(c) Der/die Autor(en) 2020

\section{A. Strzelczyk • F. C. Schmitt \\ 100 Jahre Dieter Janz}

\section{Zusammenfassung}

Am 20.04.2020 jährt sich zum 100. Mal der Geburtstag von Dieter Janz. Ihm zu Ehren erscheint dieses Heft der Zeitschrift für Epileptologie mit dem Ziel, das Wirken von Dieter Janz der letzten fünf Jahrzehnte nachzuzeichnen und neue Erkenntnisse zu dem nach ihm benannten JanzSyndrom (juvenile myoklonische Epilepsie) zusammenzufassen.

\section{0 years Dieter Janz}

\section{Abstract}

The 20 April 2020 marks the centenary of Dieter Janz's birth. This issue of Zeitschrift für Epileptologie is published in his honor with the aim of tracing the work of Dieter Janz over the last five decades and summarizing new findings on the Janz syndrome (Juvenile Myoclonic Epilepsy), which is named after him.

le Prognose beim Janz-Syndrom entwickelt und ob es durch bestimmte Persönlichkeitsmerkmale beeinflusst wird, wesentlich. Die verfügbaren Studien zum psychosozialen Outcome beim Janz-Syndrom werden von Felix von Podewils aus Greifswald unter Zusammenarbeit mit Carol Camfield aus Halifax (Kanada) zusammengefasst [33]. Neben dem bereits genannten Janz-Archiv mit Heidelberger und West-Berliner Patienten wird auch auf Langzeitendaten einer Kohorte aus Greifswald zurückgegriffen, in der seit den 70er Jahren Patienten mit JME und anderen genetischen generalisierten Epilepsien aus der DDR und spätervor allem Mecklenburg-Vorpommern über Jahrzehnte hinweg untersucht worden sind $[34,35]$.

Karl-Friederich Masuhr aus Zell an der Mosel arbeitete bereits in Heidelberg mit Dieter Janz zusammen und folgte ihm nach Berlin als Oberarzt. Er war - mit Walter Christe aus Berlin als Assistent - in der Charlottenburger Klinik in den 70erJahren für die Etablierung des VideoEEG-Monitorings zuständig. In seinem 


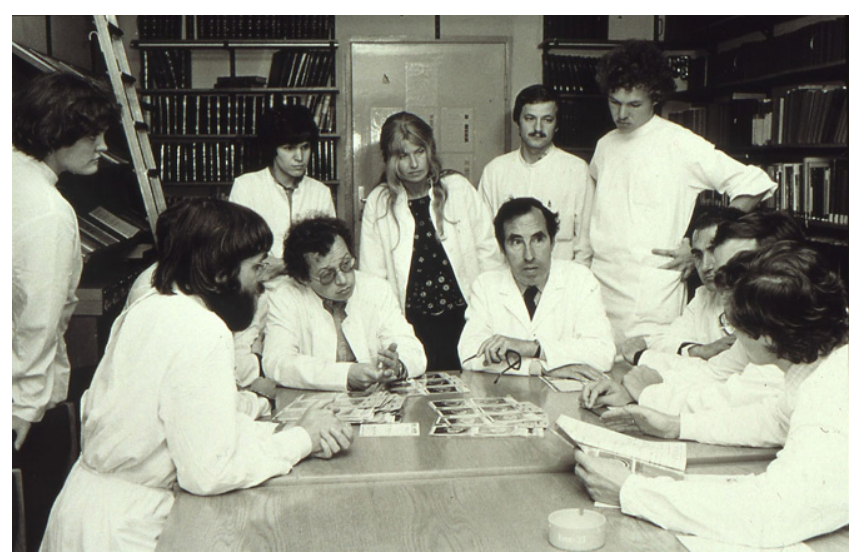

Abb. 2 ム Mittagsbesprechung in der Bibliothek 1975. (Foto: Walter Christe, Berlin)

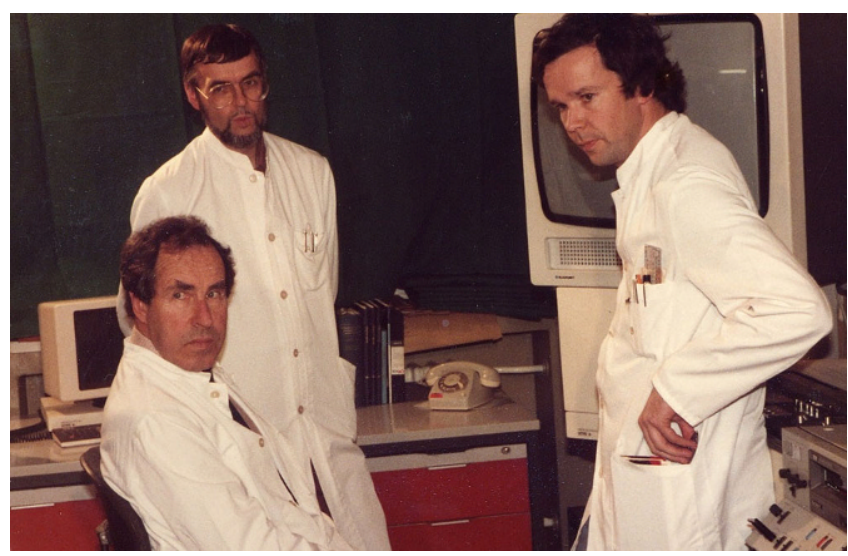

Abb. $4 \Delta$ Dieter Janz, Peter Wolf und Walter Christe im „Video-EEG-Labor". (Foto: Walter Christe, Berlin)

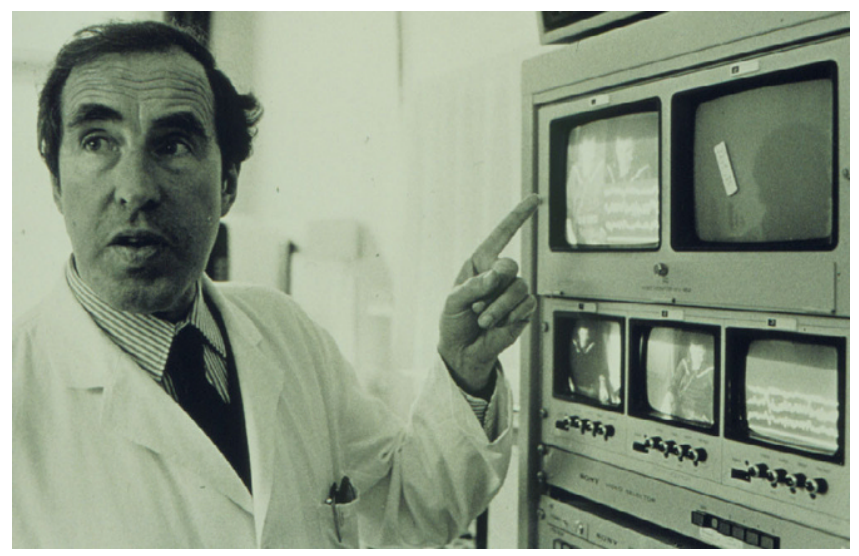

Abb. 3 ॥ Dieter Janz (1985) bei der Videoanalyse mit Zuhörern. (Foto: Walter Christe, Berlin)

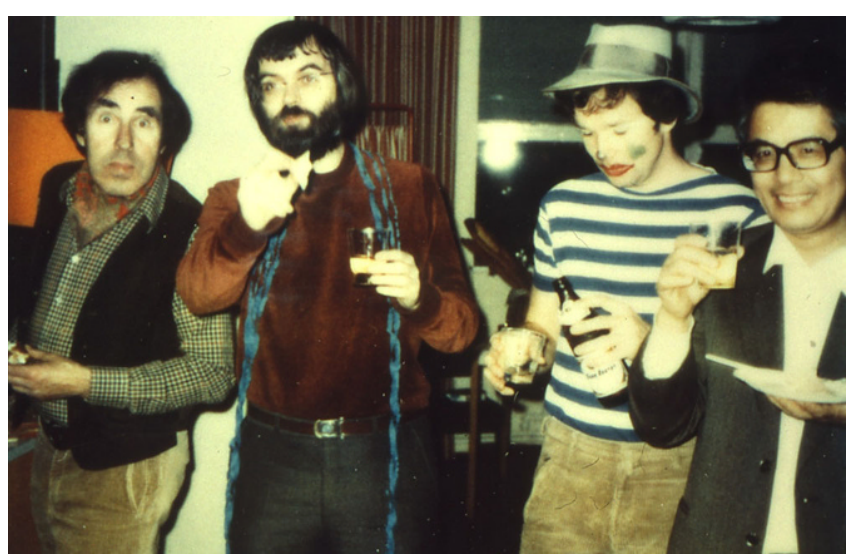

Abb. 5 \ Persönlicher Bildbeitrag von Walter Christe: „Im Haus 18 im Klinikum Charlottenburg gab es nicht nur Überlebenskämpfe bei den strengen Chefvisiten und harte Diskussionen in den zahlreichen Seminaren, es wurde auch viel und ausgiebig gefeiert. Hier Fasching 1979 mit Dieter Janz, Peter Wolf, Walter Christe und einem japanischer Gast." (Foto und Text: Walter Christe, Berlin)
Artikel beschreibt er sehr anschaulich, mit welchen Mitteln phänomenologische Aussagen unter Zuhilfenahme von einer simultanen Video-EEG Ableitung getroffen wurden [13]. Diese Untersuchungstechnik, die heute selbstverständlich ist und die eine große Datenaufarbeitung erlaubt, war in der klinischen Zeit von Dieter Janz eine neue Errungenschaft, die auch zu der syndromatologischen Zuordnung verschiedener Epilepsiesyndrome wesentlich beigetragen hat. Eine Buchbesprechung von Karl-Friederich Masuhr's Buches „Ärzte, Dichter \& Rebellen. Psychosomatische Aspekte ihres Wirkens" erschien letztes Jahr in der Zeitschrift für Epileptologie [36]. Der Themenkomplex „Psychosomatik“, „Künstler“ und „ärztliche Tätigkeit“ kann auch als eine Wei- terentwicklung des Janz'schen Wirkens gesehen werden.

Jan Rémi und Soheyl Noachtar aus München berichten über den aktuellen Stand zu EEG-Befunden beim Janz-Syndrom und anderen genetisch generalisierten Epilepsien [37]. Christian Vollmar aus München sowie Britta Wandschneider und Matthias Koepp aus London (Vereinigtes Königreich) widmen sich der Frage, welche Besonderheiten in der Bildgebung beim JanzSyndrom vorliegen [38]. Diese klinisch orientierten Artikel zum Janz-Syndrom sind v. a. als ein Update gedacht, da in der Zeitschrift für Epileptologie schon immer Beiträge zu den genetischen generalisierten Epilepsien eine wichtige Rolle spielten [39-45].
Die Nachwuchsförderung war Dieter Janz ein großes Anliegen, und so freute er sich, als 2015 auf Initiative der jungen Epileptologen ein Nachwuchspreis nach ihm benannt und durch die Prof. Dr. Peter \& Jytte Wolf - Stiftung gefördert wurde $[17,46]$. Dieter Janz hat der Benennung des Preises zu Lebzeiten zugestimmt und nahm zusammen mit Peter Wolf die erste Preisverleihung bei der Jahrestagung der Deutschen Gesellschaft für Epileptologie in Jena 2016 vor (• Abb. 10).

Sehr erfreut sind wir, diese vielen Beiträge zusammengetragen $\mathrm{zu}$ haben. Einige werden noch in den Heften 3 und 4 dieses Jahres folgen, u. a. freuen wir uns sehr auf einen Beitrag zu bislang teilweise unveröffentlichten Vorträgen von Dieter Janz, die von Bettina Schmitz aus 


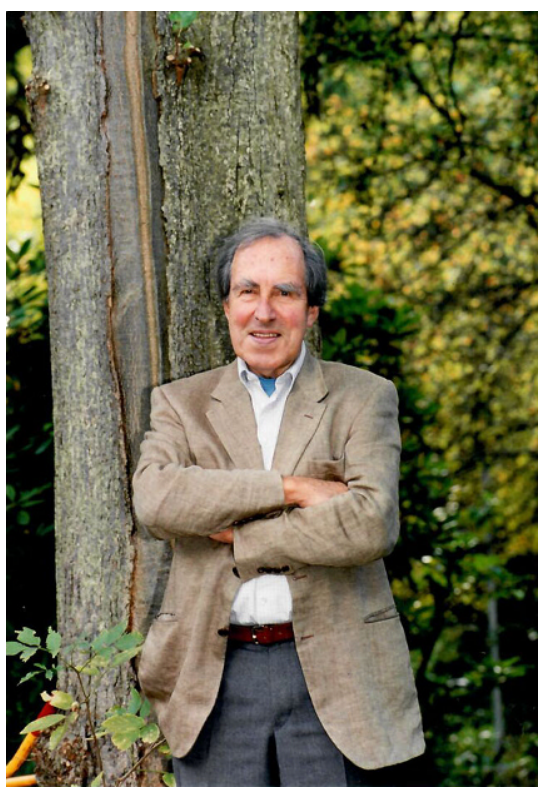

Abb. 6 A Im Garten des Hauses derFamilie Janz, in dem auch Stiftungsratssitzungen der Stiftung Michael stattfanden. (Foto: Anna Katharina Schulz, Berlin)

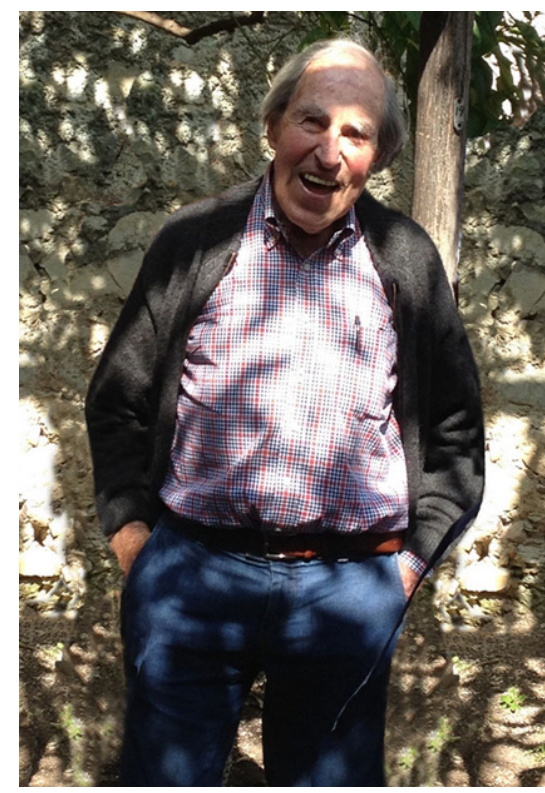

Abb. 7 \ Persönlicher Bildbeitrag von Felix Rosenow: „Dieter Janz beim Abschluss des 27. Praxisseminars in Gargnano am 13.05.2015. Er führt durch eine Limonaia am südlichen Eingang in die Stadt. Soeben hat er auf das beträchtliche Alter eines wohl etwa 100-jährigen Zitronenbaumes hingewiesen und freut sich nun über die Anmerkung, dass ja beide - er und der Baum - um die 100 Jahre alt seien." (Foto und Text:Felix Rosenow, Frankfurt am Main)

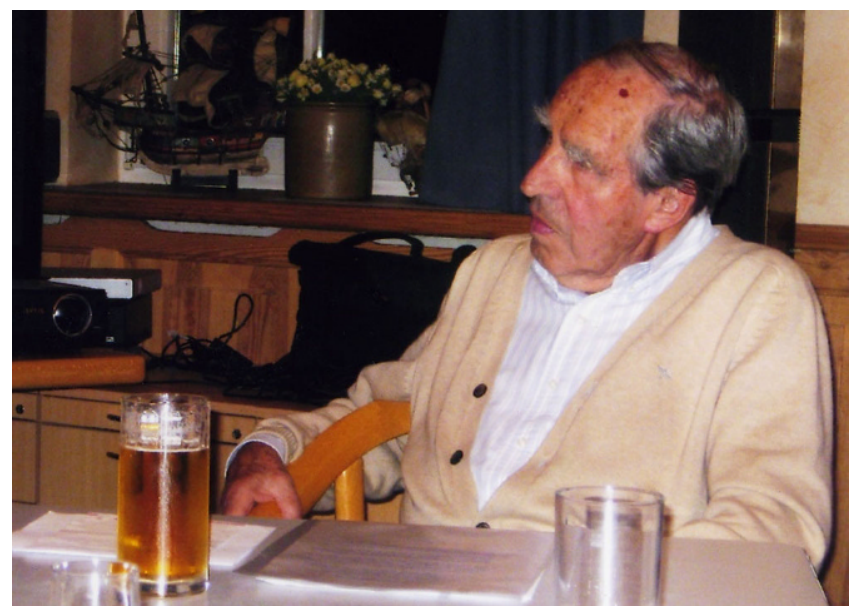

Abb. 8 A Persönlicher Bildbeitrag von Wilhelm Rimpau: „Über 30 Jahre traf sich im Hause Janz der Lesekreis der Viktor-von-Weizsäcker Gesellschaft. Zum Ritual gehörte, dass im Anschluss einer zweistündigen Leserunde und Diskussion Forster Riesling kredenzt wurde, Dieter Janz' Lieblingswein seit uralten Heidelberger Tagen. Zum Ritual der Leserunden gehören auch jährliche Ausflüge in die Berliner Umgebung über 3 Tage. Hier ein Foto von einem Treffen im Kloster Lehnin Januar 2002. Dieter Janz trinkt hier - statt seinen geliebten Wein - einmal ein Bier." (Foto und Text: Wilhelm Rimpau, Berlin)

Berlin zusammengestellt werden. Gerne können noch weitere freie Beiträge bis Anfang September 2020 eingereicht werden.

Zudem finden Sie in diesem Heft einen Nachruf auf Professor Dieter Schmidt, der einer der fünf Mitarbeiter war, die Dieter Janz nach Berlin folgten und der Ende der Achtziger ordentlicher Professor und Ordinarius für Neurologie in Berlin wurde [16]. Der Nachruf ist verfasst von Günter Krämer, Wolfgang Löscher aus Hannover, Christian Elger aus Bonn und Matti Sillanpää aus Turku (Finnland).

Günter Krämer reichte - ebenfalls ein glücklicher Umstand - eine Buchbesprechung von Hans-Wolfgang Kölmels Erfahrungsbericht als Leiter der Klinik für Neurologie der Charité Anfang der Neunziger, der einen spannenden Einblick in die Charité zur Wendezeit gibt [15].

Das Thema einer umfassenden, die Biografie des Patienten integrierenden Anamneseerhebung, deren Integration in das Verständnis der Krankheitsentwicklung und sich daraus entwickelnde diagnostische und therapeutische Konsequenzen waren auch Teil des Konzeptes in der Janz'schen Klinik. Mitarbeiter von Dieter Janz - die bereits erwähnten Mechthilde Kütemeyer, Karl-Friederich Masuhr und Wilhelm Rimpau sowie Ulrich Schultz-Venrath haben auch in der Zeitschrift für Epileptologie zu diesem Thema veröffentlicht [12, 14]. Psychosomatische Aspekte sind weiterhin ein wichtiger Bestandteil in der Epileptologie. Das nächste Heft wird sich unter der Gastherausgeberschaft von Christian Brandt aus Bielefeld und Christoph Kurth aus Kork dem Thema „Psychosomatik in der Epileptologie" widmen.

Wir möchten uns ganz herzlich bei allen Autorinnen und Autoren für ihre Beiträge zu diesem Heft und bei Familie Janz, Heinz Bühler, Walter Christe, Wilhelm Rimpau, Bettina Schmitz und Rupprecht Thorbecke bedanken, die uns bei der Erstellung der Artikel und dem Heraussuchen der Zeitzeugnisse tatkräftig unterstützt haben. Ein ganz besonderer Dank geht an Frau Bettina Knapp, die 2020 die Betreuung der Zeitschrift für Epileptologie beim Springer-Verlag über- 


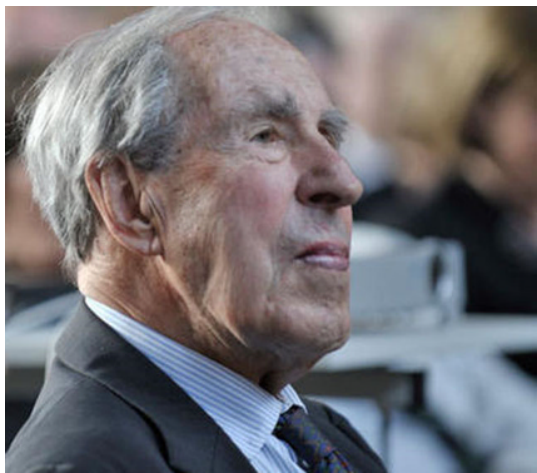

Abb. $9 \Delta$ Akademische Feier zum 90. Geburtstag. (Foto: Walter Christe, Berlin)

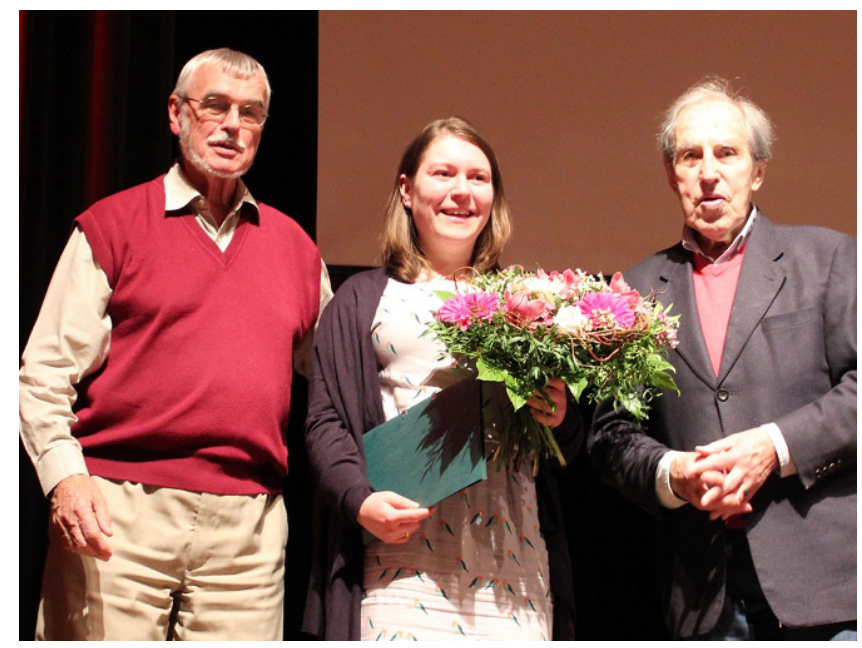

Abb. $10<$ Dieter Janz bei der ersten Verleihung des nach inm benannten Dieter-Janz-Preises an Janna Riechmann aus Marburg am 04.03.2016 in Jena. Das Preisgeld wird von der Prof. Dr. Peter und Jytte Wolf Stiftung für Epilepsie zur Verfügung gestellt. (Foto: Conventus, Jena) nommen hat und geduldig und motiviert unsere Vorschläge und Wünsche für das Jubiläumsheft umsetzte. Wie der Zufall es will, wird dieses Heft am 20.04.2020, dem 100. Geburtstag von Dieter Janz, vom Springer-Verlag zum Druck freigegeben.

Wir wünschen Ihnen viel Freude bei der Lektüre und bleiben Sie alle gesund.

Ihre

Adam Strzelczyk, Friedhelm C. Schmitt

\section{Korrespondenzadresse}

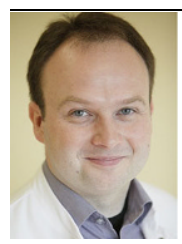

Prof. Dr. Adam Strzelczyk

Epilepsiezentrum

Frankfurt Rhein-Main,

Zentrum der Neurologie

und Neurochirurgie,

Goethe-Universität und

Universitätsklinikum

Frankfurt

Schleusenweg 2-16,

60528 Frankfurt am Main,

Deutschland

strzelczyk@med.uni-

frankfurt.de

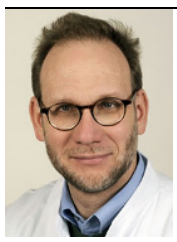

PD Dr. Friedhelm C. Schmitt Universitätsklinik für Neurologie, Otto-vonGuericke-Universität Leipziger Str. 44, 39120 Magdeburg, Deutschland fc.schmitt@med.ovgu.de

Danksagung. Herr Volker Schöwerling hat bei der Bildbearbeitung des Fotos von Walter Rimpau und Herr Jürgen Reif bei der Bildbearbeitung des Fotos von Felix Rosenow geholfen.
Interessenkonflikt. A. Strzelczyk und F.C. Schmitt geben an, dass kein Interessenkonflikt besteht.

Open Access. Dieser Artikel wird unter der Creative Commons Namensnennung 4.0 International Lizenz veröffentlicht, welche die Nutzung, Vervielfältigung, Bearbeitung, Verbreitung und Wiedergabe in jeglichem Medium und Format erlaubt, sofern Sie den/die ursprünglichen Autor(en) und die Quelle ordnungsgemäß nennen, einen Link zur Creative Commons Lizenz beifügen und angeben, ob Änderungen vorgenommen wurden.

Die in diesem Artikel enthaltenen Bilder und sonstiges Drittmaterial unterliegen ebenfalls der genannten Creative Commons Lizenz, sofern sich aus der Abbildungslegende nichts anderes ergibt. Sofern das betreffende Material nicht unter der genannten Creative Commons Lizenz steht und die betreffende Handlung nicht nach gesetzlichen Vorschriften erlaubt ist, ist für die oben aufgeführten Weiterverwendungen des Materials die Einwilligung des jeweiligen Rechteinhabers einzuholen.

Weitere Details zur Lizenz entnehmen Sie bitte der Lizenzinformation auf http://creativecommons.org/ licenses/by/4.0/deed.de.

\section{Literatur}

1. Watts G (2017) Obituary Wolfgang Dietrich Janz. Lancet 389(10076):1292

2. Wolf $P$ (2017) Nachruf auf Professor emeritus Dr. med. Wolfgang Dietrich Janz. Z Epileptol 30(1):79-80. https://doi.org/10.1007/s10309017-0105-6

3. Wolf $P$ (2020) Wer war eigentlich Dieter Janz? Z Epileptol. https://doi.org/10.1007/s1030910020-00326-10308

4. Bühler H (2020) Dieter Janz und die Stiftung Michael. Z Epileptol. https://doi.org/10.1007/ s10309-10020-00316-w

5. Janz D (1986) Epilepsy, viewed metaphysically: an interpretation of the biblical story of the epileptic boy and of Raphael's transfiguration. Epilepsia 27(4):316-322. https://doi.org/10.1111/j.1528 1157.1986.tb03548.x

6. WiedebachH(2020) Kranksein in derEpileptologie. Dieter Janz' "Nebensachen“. Z Epileptol. https:// doi.org/10.1007/s10309-10020-00327-10307
7. JanzD, Christian W (1957) Impulsiv-Petitmal.Dtsch ZNervenheilkd 176:346-386

8. Kasteleijn-Nolst Trenité DGA, Schmitz B, Janz D, Delgado-Escueta AV, Thomas $\mathrm{P}$, Hirsch $\mathrm{E}$, Lerche $\mathrm{H}$, Camfield C, Baykan B, Feucht M, Martínez-JuárezIE, Duron RM, Medina MT, Rubboli G, Jerney J, Hermann B, Yacubian E, Koutroumanidis $M$, Stephani U, Salas-Puig J, Reed RC, Woermann $F_{\text {, }}$ Wandschneider B, Bureau M, Gambardella A, Koepp MJ, Gelisse P, Gurses C, Crespel A Nguyen-Michel VH, Ferlazzo E, Grisar T, Helbig I, Koeleman BPC, Striano P, Trimble M, Buono R, Cossette P, Represa A, Dravet C, Serafini A, Savic Berglund I, Sisodiya SM, Yamakawa K, Genton P (2013) Consensus on diagnosis and management of JME: From founder's observations to current trends. Epilepsy \& Behavior 28:S87-S90. https:// doi.org/10.1016/j.yebeh.2012.11.051

9. Krämer G (2020) Zur Geschichte des JanzSyndroms (juvenile myoklonische Epilepsie). Z Epileptol. https://doi.org/10.1007/s10309-02000319-7

10. Janz D, Christian W (2005) Faksimile. Z Epileptol 18(2):50-70. https://doi.org/10.1007/s10309005-0116-6

11. Bauer J, Rimpau W (2005) Janz und das JanzSyndrom. Z Epileptol 18(2):49-49. https://doi.org/ 10.1007/s10309-005-0126-4

12. Rimpau W, Neuwirth A (2005) Wie lernen Medizinstudierende die Grundlagen der Epileptologie? Z Epileptol 18(1):23-28. https://doi.org/10.1007/ s10309-005-0102-Z

13. Masuhr KF (2020) Das Janz-Syndrom - Phänomenologie und Video-EEG-Analyse. Zeitschrift Für Epileptol. https://doi.org/10.1007/s10309-02000323-x

14. Kütemeyer $M$, Masuhr KF, Schultz-Venrath U (2005) Kommunikative Anfallsunterbrechung - Zum ärztlichen Umgang mit Patienten im Status pseudoepilepticus. Z Epileptol 18(2):71-77. https://doi.org/10.1007/s10309-005-0124-6

15. Krämer G (2020) Charité 91. Schritte in eine neue Zeit. Z Epileptol. https://doi.org/10.1007/s1030910020-00330-y

16. Krämer G, Löscher W, Elger CE, Sillanpää M (2020) Nachruf auf Professor Dr. med. Dieter Schmidt (28.04.1947-08.09.2019).Z Epileptol. https://doi. org/10.1007/s10309-020-00314-y

17. Wolf $P$ (2017) Laudatio anlässlich der Verleihung des Dieter-Janz-Preises 2017 an Frau Lara Kay. Z Epileptol 30(3):237-238. https://doi.org/10. 1007/s10309-017-0132-3 
18. Coban I, Thorbecke R (2013) Nachruf Gisela Schüler, * 07.08.1928, † 28.07.2013. Z Epileptol 26(4):255-256. https://doi.org/10.1007/s10309013-0349-8

19. Thorbecke R (2020) Sozialmedizin und sozialmedizinisches Handeln bei Dieter Janz. Z Epileptol. https://doi.org/10.1007/s10309-10020-00322-y

20. Thorbecke $R$ (2017) Rehabilitation als konstituierendes Moment der epileptologischen Arbeit von Dieter Janz. Z Epileptol 30(4):295-299. https://doi. org/10.1007/s10309-017-0125-2

21. Sander T, Hildmann T, Janz D, Wienker TF, Neitzel H, Bianchi A, Bauer G, Sailer U, Berek K, Schmitz B, Beck-Mannagetta G (1995) The phenotypic spectrum related to the human epilepsy susceptibility gene "EJM1". Ann Neurol 38(2):210-217. https:// doi.org/10.1002/ana.410380213

22. Sander T, Hildmann T, Wienker TF, Ramel $C$, Beck-Mannagetta G, Bianchi A, Sailer U, Berek K, Bauer G, Neitzel H, Schmitz B, Durner M, Johnson KJ, Janz D (1996) Common subtypes of idiopathic generalized epilepsies: Lack of linkage to D20S19 close to candidate loci (EBN1, EEGV1) on chromosome 20. Am J Med Genet 67(1):31-39. https://doi.org/10.1002/(SICI)10968628(19960216)67:1<31::AID-AJMG5>3.0.CO;2$\mathrm{V}$

23. Sander T, Bockenkamp B, Hildmann T, Blasczyk R, Kretz R, Wienker TF, Volz A, Schmitz B, BeckMannagetta G, Riess O, Epplen JT, Janz D, Ziegler A (1997) Refined mapping of the epilepsy susceptibility locus EJM1 on chromosome 6. Neurology 49(3):842-847. https://doi.org/10. 1212/wnl.49.3.842

24. Meencke HJ, Janz D, Cervos-Navarro J (1981) Neuropathology of primary generalized epilepsies with awakening grand mal. Acta Neuropathol Suppl 7:378-380. https://doi.org/10.1007/978-3642-81553-9 108

25. Meencke HJ, Janz D (1984) Neuropathological findings in primary generalized epilepsy: a study of eight cases. Epilepsia 25(1):8-21. https://doi.org/ 10.1111/j.1528-1157.1984.tb04149.x

26. Scheffer IE, Berkovic S, Capovilla G, Connolly MB, French J, Guilhoto L, Hirsch E, Jain S, Mathern GW, Moshé SL, Nordli DR, Perucca E, Tomson T, WiebeS, Zhang YH, Zuberi SM (2017) ILAE classification of the epilepsies: Position paper of the ILAE Commission for Classification and Terminology. Epilepsia 58(4):512-521. https://doi.org/10.1111/ epi.13709

27. Scheffer IE, Berkovic S, Capovilla G, Connolly MB, French J, Guilhoto L, Hirsch E, Jain S, Mathern GW, Moshé SL, Nordli DR, Perucca E, Tomson T, Wiebe S, Zhang YH, Zuberi SM (2018) ILAE-Klassifikation der Epilepsien: Positionspapier der ILAE-Kommission für Klassifikation und Terminologie. Z Epileptol 31(4):296-306. https://doi.org/10.1007/s10309018-0218-6

28. Vorderwülbecke B, Holtkamp M (2020) Langzeitanfallsprognose bei genetischen generalisierten Epilepsien. Z Epileptol. https://doi.org/10.1007/ s10309-020-00317-9

29. Holtkamp M, Janz D, Kirschbaum A, Kowski $A B$, Vorderwulbecke BJ (2018) Absence epilepsy beyond adolescence: an outcome analysis after 45 years of follow-up. J Neurol Neurosurg Psychiatry 89(6):603-610. https://doi.org/10.1136/jnnp2017-317052

30. Vorderwulbecke BJ, Kowski AB, Kirschbaum A, Merkle H, Senf P, Janz D, Holtkamp M (2017) Longterm outcome in adolescent-onset generalized genetic epilepsies. Epilepsia 58(7):1244-1250. https://doi.org/10.1111/epi.13761
31. Senf P, Schmitz B, Holtkamp M, Janz D (2013) Prognosis of juvenile myoclonic epilepsy 45 years after onset: Seizure outcome and predictors. Neurology 81(24):2128-2133. https://doi.org/10. 1212/01.wnl.0000437303.36064.f8

32. Kurlemann G, Krämer G (2020) Absetzen von Antikonvulsiva beim Janz-Syndrom (Juvenile myoklonische Epilepsie) in der Neuropädiatrie: Pro und contra. Z Epileptol. https://doi.org/10.1007/ s10309-10020-00320-10300

33. von Podewils F (2020) Psychosocial outcome in juvenile myoclonic epilepsy (Janz syndrome) and other genetic generalized epilepsies. Z Epileptol. https://doi.org/10.1007/s10309-020-00318-8

34. Geithner J, Schneider F, Wang Z, Berneiser J, Herzer R, Kessler C, Runge U (2012) Predictors for long-term seizure outcome in juvenile myoclonic epilepsy: 25-63 years of follow-up. Epilepsia 53(8):1379-1386. https://doi.org/10.1111/j.15281167.2012.03526.x

35. Schneider-von Podewils F, Gasse C, Geithner J, WangZI, Bombach P, Berneiser J, HerzerR, KesslerC, Runge U (2014) Clinical predictors of the longterm social outcome and quality of life in juvenile myoclonic epilepsy: 20-65 years of follow-up. Epilepsia 55(2):322-330. https://doi.org/10.1111/ epi.12491

36. Oswalt S (2019) Buchbesprechung. Z Epileptol 32(3):248-249. https://doi.org/10.1007/s10309019-0278-2

37. Rémi J, Noachtar S (2020) EEG bei Janz-Syndrom (juvenile myoklonische Epilepsie) und anderen generalisierten Epilepsien. Z Epileptol. https://doi. org/10.1007/s10309-020-00325-9

38. Vollmar C, Wandschneider B, Koepp M (2020) Bildgebung beim Janz-Syndrom (juvenile myoklonische Epilepsie). Z Epileptol. https://doi.org/10. 1007/s10309-020-00324-w

39. Marquetand J, Lerche H (2018) Besondere Bildgebung bei sonstigen genetisch determinierten Epilepsien. Z Epileptol 31(2):148-151. https://doi. org/10.1007/s10309-017-0167-5

40. Wandschneider B, Vollmar C (2018) Besondere bildgebende Befunde bei primär generalisierten Epilepsien. Z Epileptol 31(2):144-147. https://doi org/10.1007/s10309-018-0179-9

41. Wandschneider B, Vollmar C, Woermann FG, Koepp MJ (2012) Bildgebung bei idiopathisch generalisiertenEpilepsien.ZEpileptol 25(3):194-199. https://doi.org/10.1007/s10309-012-0259-1

42. Rémi J, Stoyke C, Noachtar S (2008) Die juvenile myoklonische Epilepsie wird häufig inadäquat behandelt. Z Epileptol 21(1):2-5. https://doi.org/ 10.1007/s10309-008-0290-4

43. Winter N, Muhle H, Lerche H (2016) Idiopathische generalisierte Epilepsien und Fotosensitivität. Z Epileptol 29(2):63-69. https://doi.org/10.1007/ s10309-015-0033-2

44. von Podewils F, Geithner J (2015) Idiopathisch generalisierte Epilepsien. Z Epileptol 28(2):129-133. https://doi.org/10.1007/s10309-015-0422-6

45. Weber YG, Sander T, Lerche H (2011) Idiopathische generalisierte Epilepsien. Z Epileptol 24(2):100-107. https://doi.org/10.1007/s10309011-0168-8

46. Schubert-Bast S (2018) Laudatio anlässlich der Verleihung des Dieter-Janz-Preises 2018 an Dr. med. Barbara Schmeiser. Z Epileptol 31(3):214-215. https://doi.org/10.1007/s10309018-0198-6
Genspezifische Therapien neurologischer Erkrankungen

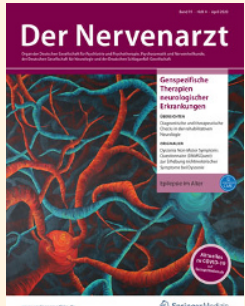

Genspezifische Ansätze, die entweder das Krankheitsgen selbst oder einen Krankheitsmechanismus korrigieren, werden die Behandlung neuro-

logischer Erkrankungen nachhaltig verändern. Mit dem Leitthema in Der Nervenarzt 04/2020 wollen wir Ihnen einen Überblick über die immensen Chancen, aber auch Herausforderungen der neuen genspezifischen Therapien bei ausgewählten neurologischen Erkrankungen vermitteln.

- Genspezifische Therapieansätze bei amyotropher Lateralsklerose in Gegenwart und Zukunft

- Neue Therapien der spinalen Muskelatrophie

- Genselektive Therapieansätze bei der Huntington-Krankheit

- Genspezifische Therapieansätze bei der Alzheimer-Krankheit und anderen Tauopathien

- Genspezifische Therapieansätze bei Muskelerkrankungen

\section{Suchen Sie noch mehr zum Thema?}

Mit e.Med - den maßgeschneiderten Fortbildungsabos von Springer Medizin - haben Sie Zugriff auf alle Inhalte von SpringerMedizin.de. Sie können schnell und komfortabel in den für Sie relevanten Zeitschriften recherchieren und auf alle Inhalte im Volltext zugreifen.

Weitere Infos zu e.Med finden Sie auf springermedizin.de unter „Abos" 